\title{
Dank Interventionsbündel mehr Sicherheit bei Blasenkathetern
}

\author{
Stephanie Züllig ${ }^{a}$, Jonas Marschall ${ }^{b}$ \\ ${ }^{a}$ Dr. sc. nat., Programmleiterin progress! Sicherheit bei Blasenkathetern, Patientensicherheit Schweiz \\ b PD Dr. med., Leiter Surveillance Blasenkatheter, Swissnoso
}

\begin{abstract}
Wie internationale Verbesserungsprogramme gezeigt haben, kann der Einsatz von Blasenkathetern im Spital mittels eines evidenzbasierten Interventionsbündels erfolgreich reduziert werden. An die lokalen Verhältnisse angepasst lässt sich das Modell erfolgreich auf die Schweiz übertragen. Dies zeigen die Ergebnisse des nationalen Pilotprogramms «progress! Sicherheit bei Blasenkathetern».
\end{abstract}

"progress! Sicherheit bei Blasenkathetern" ist das dritte nationale Pilotprogramm, das im Rahmen der Qualitätsstrategie des Bundes im schweizerischen Gesundheitswesen durchgeführt wurde. Die Stiftung Patientensicherheit Schweiz und Swissnoso haben das Programm gemeinsam entwickelt und in den Jahren 2016-2018 umgesetzt. Das Bundesamt für Gesundheit (BAG) hat es finanziert. Das Programm hatte zum Ziel, die Verwendung von Blasenkathetern zu reduzieren

\section{Flankiert von einer Sensibilisierungskampagne implementierten sieben Pilotspitäler ein evidenzbasiertes Interventionsbündel.}

und damit die Häufigkeit von Katheter-bedingten Komplikationen wie Harnwegsinfektionen oder Traumata von Harnröhre und -blase zu senken. Dies vor dem Hintergrund, dass transurethrale Dauerkatheter, hier Blasenkatheter genannt, im Spitalalltag verbreitet zur Anwendung kommen, jedoch häufig ohne Indikation und ausreichende Notwendigkeit [1, 2].

Flankiert von einer Sensibilisierungskampagne implementierten sieben Pilotspitäler ein evidenzbasiertes Interventionsbündel, bestehend aus den folgenden

\section{Résumé}

Comme l'ont montré les programmes d'amélioration internationaux, l'utilisation de sondes vésicales à l'hôpital peut être réduite avec succès, grâce à un faisceau d'interventions basé sur des données probantes. Adapté aux conditions locales, le modèle peut tout à fait être appliqué à la Suisse. C'est ce que montrent les résultats du programme pilote national «progress! La sécurité dans le sondage vésical». drei Massnahmen: 1) Indikationsliste, 2) tägliche Überprüfung der Notwendigkeit des Katheters (Re-Evaluation) und 3) Schulung des Personals zum sicheren Umgang mit Blasenkathetern. Um die Wirksamkeit des Interventionsbündels aufzuzeigen, erhoben die Pilotspitäler vor und nach Implementierung der Massnahmen im Rahmen der Surveillance während je dreier Monate Daten zur Katheternutzung und zu den damit einhergehenden Katheter-assoziierten Harnwegsinfektionen (Catheter-Associated Urinary Tract Infections, CAUTI) sowie zu verschiedenen nicht-infektiösen Komplikationen. Mittels Prozessvariablen wurden darüber hinaus der Anteil indizierter Katheter und die durchgeführten Re-Evaluationen erfasst. Eine Mitarbeiterbefragung untersuchte, ob sich Wissen und Einstellungen im Umgang mit Blasenkathetern im Verlauf des Pilotprogramms änderten.

\section{Weniger Katheter, häufigere Überprüfung, weniger Komplikationen}

Wie die Auswertungen zeigen, ging die Katheternutzung in den Pilotspitälern signifikant von 23,7 auf 21,0\% zurück. Der Anteil der Blasenkatheter mit einer dokumentierten Indikation erhöhte sich signifikant von 74,5 auf 90,0\%. Auch die Anzahl Überprüfungen, ob die Notwendigkeit des Katheters gemäss Indikationsliste noch gegeben ist, stieg signifikant von 168 auf 624 Re-Evaluationen/1000 Kathetertage. Die Inzidenz von CAUTI war in den Pilotspitälern von Beginn an niedrig und änderte sich über die Dauer des Programms nicht (0,02 Infekte/100 Kathetertage). Auch die Rate der Kathetertage blieb konstant und bewegte sich zwischen 17,4 (Vorher-Messung) und 13,5 (Nachher- 
Messung) Kathetertage/100 Patiententage. Hingegen ging die Rate nicht-infektiöser Komplikationen, wie Makrohämaturie oder Blutungen aus der Harnröhre, signifikant von 0,8 auf 0,6 Komplikationen/100 Patiententage zurück.

\section{Mehr Wissen und veränderte Einstellungen bei Ärzteschaft und Pflegepersonal}

Die Mitarbeitenden konnten nach Implementierung des Interventionsbündels mehr Wissensfragen korrekt beantworten. Zudem schätzten sie den Umgang mit Blasenkathetern innerhalb ihrer Organisation als restriktiver und sicherer ein. Dies liess sich beispielsweise an der gestiegenen Zustimmung zur Aussage "Blasenkatheter auf der Abteilung werden nur als klar indizierte medizinische Massnahme gelegt» oder «die tägliche Überprüfung, ob ein Blasenkatheter noch notwendig ist, ist bei uns selbstverständlich" ablesen. Bei den Mitarbeitenden selbst liess sich eine erhöhte Bereitschaft feststellen, einen aktiven Beitrag zum sicheren Umgang mit Blasenkathetern zu leisten.

\section{Ein Blick hinter die Kulissen der Pilotspitäler}

Wie die Daten zeigen, hat sich das Interventionsbündel bewährt, und die Ziele des nationalen Pilotprogramms wurden erreicht. Die Pilotspitäler haben vorgeführt, dass das Interventionsbündel umsetzbar ist - sowohl im kleinen Regionalspital wie auch im grossen Universitätsspital. Der Aufwand, den sie dafür leisteten, variierte und war abhängig von den zur Verbreitung und Umsetzung des Interventionsbündels ergriffenen Massnahmen, der Art und Weise, wie die Mitarbeitenden sensibilisiert und geschult wurden (Online-Schulung, praktisches Training am Phantom) und von der
Surveillance. Zwar war das praktische Training zur Kathetereinlage mit einem beträchtlichen Organisationsaufwand verbunden, es stiess jedoch bei vielen Mitarbeitenden auf ein Bedürfnis und wurde laut Angaben der Pilotspitäler sehr geschätzt. Auch die Surveillance erwies sich als aufwendig. Insbesondere die Erhebung der symptomatischen CAUTI benötigte viel Zeit, da zur Bestätigung der Infektion nebst klinischen Symptomen auch der Nachweis von Erregern in der Urinkultur erforderlich ist. Die meisten Pilotspitäler mussten diese Informationen von Hand aus verschiedenen Dokumentationssystemen herausziehen.

\section{Was es für eine erfolgreiche Verbesserung im Umgang mit Blasenkathetern braucht}

Patientensicherheit Schweiz und Swissnoso empfehlen, das Interventionsbündel in allen Schweizer Akutspitälern zu implementieren und dessen Wirksamkeit zu monitorisieren. Die evidenzbasierte Indikationsliste stellt sicher, dass nur diejenigen Patientinnen und Patienten einen Blasenkatheter erhalten, die ihn benötigen. Mit der täglichen Re-Evaluation wird geprüft, ob die Notwendigkeit des Katheters gemäss Indikationsliste noch gegeben ist. Falls nicht, wird der Katheter entfernt. Die Personalschulung macht die Einlage und Pflege des Katheters sicherer.

Für die Einführung des Interventionsbündels ist es entscheidend, dass der Verbesserungsbedarf erkannt und der Wille zur Veränderung vorhanden sind. Wichtig ist, dass die einzelnen Massnahmen von den verantwortlichen Stellen mitgetragen und gefördert werden. Pilotspitäler, die zur Verbreitung und Umsetzung des Interventionsbündels sogenannte Champions eingesetzt haben, haben damit gute Erfahrungen gemacht und sind überzeugt, dass diese wesentlich zum Gelingen des Projekts in ihrem Spital beigetragen haben.

Tabelle 1: Indikationsliste "progress! Sicherheit bei Blasenkathetern».

\begin{tabular}{|c|c|}
\hline \multirow[t]{2}{*}{ Harnverhalt } & akuter Harnverhalt \\
\hline & symptomatische chronische Ausflussobstruktion PLUS >300 ml Restharn \\
\hline Urinmonitoring/Bilanzierung & $\begin{array}{l}\text { in regelmässigen kurzen Intervallen PLUS direkte therapeutische Konsequenzen } \\
\text { aus der Bilanzierung }\end{array}$ \\
\hline \multirow[t]{4}{*}{ Operation } & Operationsdauer $>4$ Stunden \\
\hline & perioperativ: aus operationstechnischen Gründen \\
\hline & Urogenitalchirurgie und/oder Beckenbodenchirurgie \\
\hline & epidurale/peridurale Anästhesie/Analgesie \\
\hline Dekubitalulzera PLUS Inkontinenz & Dekubitus Grad III oder IV oder Hauttransplantate sacral/perineal PLUS Inkontinenz* \\
\hline Prolongierte Immobilisation & aus medizinischen Gründen*, insbesondere zur Schmerzreduktion \\
\hline \multirow[t]{2}{*}{ Palliation PLUS Komfort } & $\begin{array}{l}\text { terminal-palliative Situation PLUS Störung der Blasenfunktion PLUS/ODER Schwierigkeit } \\
\text { einer normalen Miktion* }\end{array}$ \\
\hline & bei hohem Leidensdruck PLUS auf Wunsch des informierten Patienten \\
\hline
\end{tabular}

* Nach Ausschöpfung von Alternativmethoden zur kontrollierten Urinableitung. 
Champions sind engagierte und von ihren Kolleginnen und Kollegen geschätzte Mitarbeitende, die sich dafür einsetzen, andere von der Notwendigkeit von Verbesserungsmassnahmen zu überzeugen und zur Mitarbeit zu motivieren.

Im Vergleich mit der Surveillance des Pilotprogramms lässt sich der Aufwand für das Monitoring reduzieren. Eine Möglichkeit wäre es, sich auf die Erhebung der Katheternutzung zu konzentrieren und die Katheternutzungsrate (Kathetertage/100 Patiententage) als

\section{Im Vergleich mit der Surveillance des Pilotprogramms lässt sich der Aufwand für das Monitoring reduzieren.}

Surrogatmarker zu verwenden (d.h. ein auf Blasenkatheter bezogenes Device Stewardship) [3, 4]. Die Bestimmung der Katheternutzungsrate ist deutlich weniger aufwendig und trägt dem Umstand Rechnung, dass die Definition Katheter-assoziierter Harnwegsinfektionen auf teilweise unterschiedlichen labordiagnostischen Parametern beruht, die eine Vergleichbarkeit erschweren. Die Katheternutzungsrate kann auf relativ unkomplizierte Weise aus der Pflegedokumentation (Einlage- und Entfernungsdatum des Katheters) sowie aus dem spitalinternen Klinikinformationssystem (Ein- und Austrittsdatum) des Patienten berechnet werden. Diese Messung ist auch für Spitäler mit einem niedrigeren Automatisierungsgrad mit überschaubarem Zusatzaufwand möglich.

Ausführlichere Informationen können im Update zur Schriftenreihe Nr. 9 «Sicherheit bei Blasenkathetern» nachgelesen werden, das zum Abschluss des Pilotprogramms im September 2018 publiziert wurde. $\mathrm{Zu}$ sammen mit der Schriftenreihe bietet das Update umfangreiches Hintergrundwissen und praxiserprobte Handlungsanleitungen zur Reduktion von (unnötigen) Blasenkathetern im Akutspital [5]. Beide Dokumente sind in den Sprachen Deutsch, Französisch und Italienisch online verfügbar unter www.patientensicherheit. ch/blasenkatheter.

\section{Literatur}

1 Magill SS, Edwards JR, Bamberg W, Beldavs ZG, Dumyati G, Kainer MA, et al. Multistate point-prevalence survey of health care-associated infections. N Engl J Med. 2014;370(13):1198-208.

2 Munasinghe RL, Yazdani H, Siddique M, Hafeez W. Appropriateness of use of indwelling urinary catheters in patients admitted to the medical service. Infect Control Hosp Epidemiol. 2001 Oct;22(10):647-9.

3 Fakih MG, Gould CV, Trautner BW, Meddings J, Olmsted RN, Krein SL, et al. Beyond Infection: Device Utilization Ratio as a Performance Measure for Urinary Catheter Harm. Infect Control Hosp Epidemiol. 2016;37(3):327-33.

4 Abrantes-Figueiredo JI, Ross JW, Banach DB. Device Utilization Ratios in Infection Prevention: Process or Outcome Measure? Curr Infect Dis Rep. 2018;20(5):8

5 Züllig S, Mascherek A. Sicherheit bei Blasenkathetern. Empfehlungen im Rahmen des nationalen Pilotprogramms progress! Sicherheit bei Blasenkathetern. Zürich: Stiftung für Patientensicherheit; 2016 